\title{
Flows in active region loops observed by Hinode EIS
}

\author{
G. Del Zanna
}

\author{
Mullard Space Science Laboratory, University College London, Holmbury St. Mary Dorking RH5 6NT, UK \\ DAMTP, Centre for Mathematical Sciences, Wilberforce road Cambridge CB3 0WA, UK \\ e-mail: GDelZanna@spd.aas.org \\ Received 16 November 2007 / Accepted 14 January 2008
}

\section{ABSTRACT}

\begin{abstract}
We aim to investigate the overall characteristics of coronal active region loops and their evolution. The Hinode database was searched for observations of active regions as they crossed the Sun centre. NOAA 10926 was selected. The morphology of this young active region did not significantly change over the course of a few days. Persistent redshifts, stronger in cooler lines (about $5-10 \mathrm{~km} \mathrm{~s}^{-1}$ in Fe XII and 20-30 $\mathrm{km} \mathrm{s}^{-1}$ in Fe VIII), were observed in most loop structures with the EUV Imaging Spectrometer. Persistent blueshifts, stronger in the hotter lines (typically $5-20 \mathrm{~km} \mathrm{~s}^{-1}$ in Fe XII and $10-30 \mathrm{~km} \mathrm{~s}^{-1}$ in Fe XV), were present in areas of weak emission, in a sharp boundary between the low-lying "hot" 3 MK loops and the higher "cooler" 1 MK loops.
\end{abstract}

Key words. Sun: corona - Sun: UV radiation - Sun: activity

\section{Introduction}

It is widely accepted that measurements of flows in active regions are important as they provide constraints for the many physical models that explain the formation and the dynamics of the many structures that are present. It is well known that $\mathrm{H} \alpha$ loops continuously form and show a persistent pattern of downflows (cf. the review of Zwaan 1985). These downflows are normally measured both as Dopplershifts and directly as plasma motions. A number of papers explain the downflows of cool material in terms of catastrophic cooling caused by a significant decrease of the heating in the corona (see, e.g. Schrijver 2001).

At transition-region (TR) temperatures, there is an extensive literature that discusses measurements of dopplershifts, often thought to be the result of real plasma motions. Many theoretical models have been put forward to explain these observations. In the majority of cases, TR lines appear red-shifted. There are numerous measurements from the spectrometers aboard OSO 8, the HRTS flights, Skylab, and more recently from the the CDS and SUMER aboard SOHO. Most of the published results, however, do not discuss measurements of Dopplershifts across an entire loop or active region system as observed ondisk. A large body of literature exists on: measurements of the quiet Sun or active regions at a single slit position; general properties of flows and line profiles (many by studying center-to-limb variations); flows observed above the solar limb, where TR lines are clearly visible (without background emission), but where the footpoints of loops are difficult to identify. Spatially-resolved spectral measurements of TR line profiles on-disk do exist. Most published studies, however, have focused on measurements around sunspots (or just on sunspot plumes, where redshifts are normally observed). For example, Gurman \& Athay (1983) reported blueshifts in C IV above sunspots as observed with SMM/UVSP. Brynildsen et al. (1998) have reported Dopplershifts from SOHO CDS and SUMER lines around sunspots. As described by Winebarger et al. (2002) and Marsch et al. (2004), SUMER measurements have shown a persistent pattern of Dopplershifts in lines from the chromosphere up to the transition region (Ne VIII). Winebarger et al. (2002) found persisent redshifts in the legs of cool 1 MK loops seen in the TRACE $171 \AA$ band, in puzzling contrast to the apparent upflow of plasma seen in the same band as intensity perturbations (see, e.g. Schrijver et al. 1999). Marsch et al. (2004) found clear blue-shifts above sunspots, but it was not clear how much they were related to siphon flows in loops or to open field regions.

Measurements of Dopplershifts in "coronal" $(T>1 \mathrm{MK})$ lines in active region loops have been comparatively scarce, and share the same limitations as the above-mentioned TR measurements. Achour et al. (1995) and many other authors have reported blue-shifts in a few coronal lines obtained with single slit exposures. Others, such as Brynildsen et al. (1998), have reported Dopplershifts from CDS lines, but with small fields of view.

The Hinode EUV Imaging Spectrometer (EIS, see Culhane et al. 2007), with its two wavelength bands (SW: 163-209 A; LW: 242-289 $\AA$ ) and excellent spectral resolution, allows routine measurements of line shifts and broadenings in a wide range of lines, formed at TR to coronal (and flare) temperatures (see Young et al. 2007a; Young et al. 2007b, for a preliminary discussion of the EIS lines). Combined with its high sensitivity, large field of view and higher telemetry, EIS provides for the first time the opportunity to measure small Dopplershifts in coronal lines across an active region as a function of time. Accuracy of line-of-sight velocities for CDS coronal lines were at most 10-20 $\mathrm{km} \mathrm{s}^{-1}$, for pre-SOHO-loss spectra, while EIS can provide accuracies of a few $\mathrm{km} \mathrm{s}^{-1}$. Large field of views are important for instruments such as CDS and EIS, which lack absolute wavelength calibration, and have a spectral dispersion that is highly variable, so quiet regions need to be used to estimate reference wavelengths. Another advantage of the EIS instrument over CDS is the better spatial resolution (by a factor 
of about 2-3). Compared to SUMER, EIS has the advantage of having large count rates in coronal lines, and that all lines are simultaneously recorded.

Since its launch, EIS has shown that Dopplershifts are ubiquitous at all temperatures in all active regions. Preliminary results of EIS observations of NOAA 10926 on 2006 December 2 (Del Zanna 2007) showed a consistent pattern of Dopplershifts and non-thermal widths across the active region. The same observation was independently chosen by other authors who have focused on flow and width measurements in Fe XII (Doschek et al. 2007). The main aim of this Letter is to present successive observations of the same active region with a large field of view, and to confirm that the observed patterns are persistent over a long period of time. The EIS measurements are shown in the context of the higher-resolution TRACE EUV images, and of the underlying photospheric magnetic fields.

\section{Analysis}

The TRACE observations have been processed using the standard Solarsoft routines, while the EIS data have been analysed using user-written routines. Aside from a cosmic ray and bias removal, the main complexity in the EIS spectra is the presence of a large, not-reproduceable $\left(70 \mathrm{~km} \mathrm{~s}^{-1}\right)$ orbital variation in the two EIS bands. Real Dopplershifts in the spectral lines are sufficiently high and omni-present in active regions that it is not trivial to correct for this orbital variation. For the data presented here, average spectra in a few strong lines and in regions as far away as possible from the main flows were fitted in order to apply a correction to all the observed lines. Typical uncertainties in this correction are $5 \mathrm{~km} \mathrm{~s}^{-1}$ across an entire raster. Locally, relative Dopplershifts of $1-3 \mathrm{~km} \mathrm{~s}^{-1}$ can be measured. Reference wavelengths in the lines were obtained from quiet areas as far away as possible from the main flows. The selection of different "reference" areas typically provides a variation of $3 \mathrm{~km} \mathrm{~s}^{-1}$, which is assumed as a typical uncertainty here, until we have a better understanding of the instrument. The EIS spectral lines have an instrumental full-width-half-maximum (FWHM) of 2.5 pixels, and line profiles were found to be nearly Gaussian. Non-thermal line widths were calculated assuming the instrumental widths from the ground calibration, and a temperature of peak ion abundance in ionization equilibrium.

The co-alignment of the different datasets was achieved only when TRACE white-light, EUV and SOHO/MDI data were available, and had an uncertainty of a few arcseconds. The EIS pointing was adjusted using the TRACE $171 \AA$ band and lines in the EIS SW channel. The offset between the LW and SW channels appears to be variable, and was checked by using lines formed at similar temperatures in the two bands.

\section{Results}

NOAA 10926 was observed by Hinode for a few days as it crossed the meridian. It had typical characteristics of most young ARs. It was mainly bipolar, composed of a leading group of sunspots with a strong magnetic field concentration, followed by a number of scattered smaller areas of opposite polarity (cf. Fig. 1). During its passage across the solar disk, continuous flux emergence was observed. The first good EIS raster was obtained on 2006 November 30 during 16:25-18:40 UT, rastering an area of $256^{\prime \prime} \times 256^{\prime \prime}$ with the $1^{\prime \prime}$ slit and $30 \mathrm{~s}$ exposure (JTM002 EIS study). The second one presented here was obtained on 2006 December 1 during 19:15-22:34 UT and rastering $377 \times 256^{\prime \prime}$ (JTM004 EIS study). These studies did not include transition region lines, with the exception of the Fe VIII $(\log T[\mathrm{~K}]=5.8)$
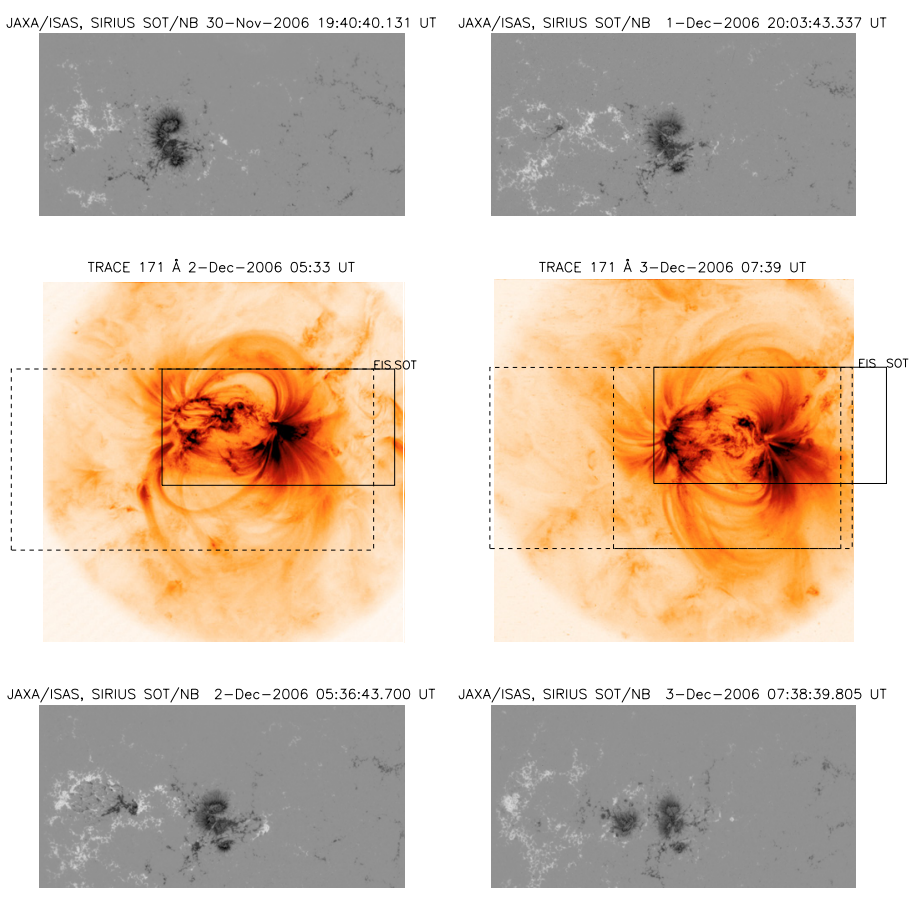

Fig. 1. SOT/NFI Stokes- $V$ negative images of AR 10926, showing the evolution of the flux emergence. TRACE $171 \AA$ negative images of NOAA 10926, with the fields of view of EIS and Stokes- $V$ images of some of the observations presented here.

$185.22 \AA$, which in the core of active regions is blended with a Ni XVI line. The Ni XVI line is in the red wing and indeed in the velocity plots it is clear that the apparent redshifts in the Fe VIII in the AR core are due to this blend (cf. Fig. 2). The other lines presented here are the self-blend Fe XII $(\log T[\mathrm{~K}]=6.1)$ $195.12 \AA$, and the (blended) resonance Fe XV $(\log T[\mathrm{~K}]=6.3)$ $284.16 \AA$ line. A few other lines from Fe X, Fe XI, Fe XIII and Fe XIV were observed but show similar characteristics. The third and fourth rasters considered here were taken during 02:5407:23 and 05:30-09:59 UT on 2006 December 2 and 3, covering a FOV of $512 \times 256^{\prime \prime}$ with the same JTM004 study. Figure 2 shows monochromatic and corresponding Dopplergram images in the selected lines obtained from the EIS rasters, with the same FOV, centred on the AR. Redshits in the line centroids have positive values. When possible, contours from the SOT/NFI Stokes$\mathrm{V}$ images are superimposed.

It is clear that the AR evolved considerably during its meridian passage; however the overall features remained unchanged. A large number of loops, emitting at different temperatures and that are physically distinct, are present. The hottest loops are in the core of the active region. At about 2-3 MK active region loops become more diffuse and disappear in the 'background' multi-thermal emission which appears unresolved at the TRACE resolution ( $\left.1^{\prime \prime}\right)$. This was already known from SOHO/CDS (cf. Del Zanna \& Mason 2003; and Del Zanna et al. 2006a). The presence of loops at different temperatures can have a profound effect when measuring Dopplershifts, considering that each line profile is an average along the line of sight.

The most striking feature is the persistence of the redshifts, more prominent in the cooler lines, in almost all loop structures. Line-of-sight redshifts in the loops are about $5-10 \mathrm{~km} \mathrm{~s}^{-1}$ in Fe XII and $20-30 \mathrm{~km} \mathrm{~s}^{-1}$ in Fe VIII near their legs, where the loop temperature decreases. Blueshifts in all lines are clearly present and persistent in the same locations, predominantly 

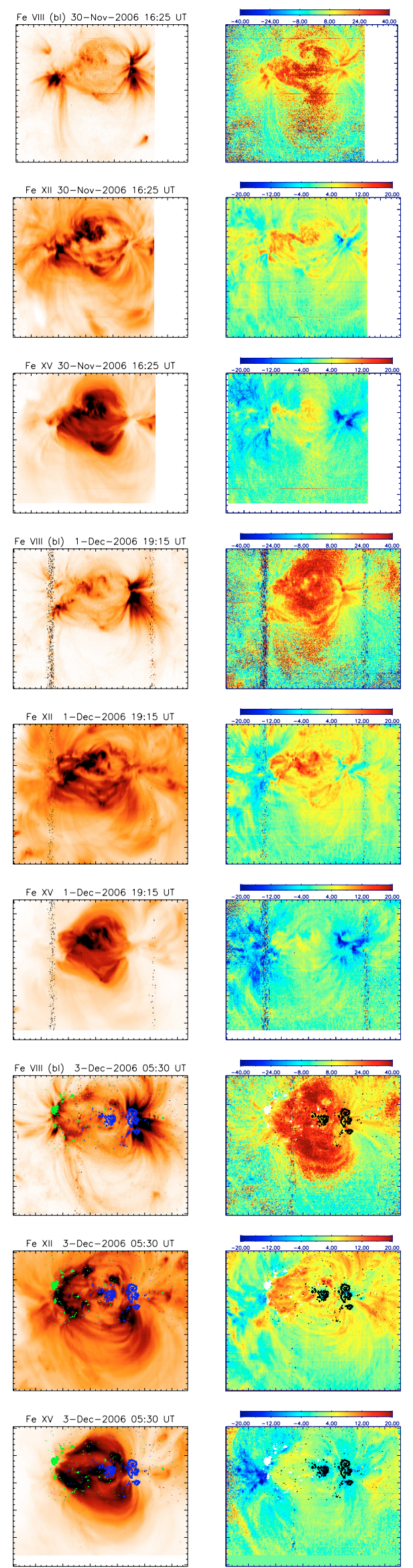

Fig. 2. Monochromatic (negative) images and dopplergrams $\left(\mathrm{km} \mathrm{s}^{-1}\right)$ of NOAA 10926 in Fe VIII, Fe XII, Fe XV as it crossed the meridian.

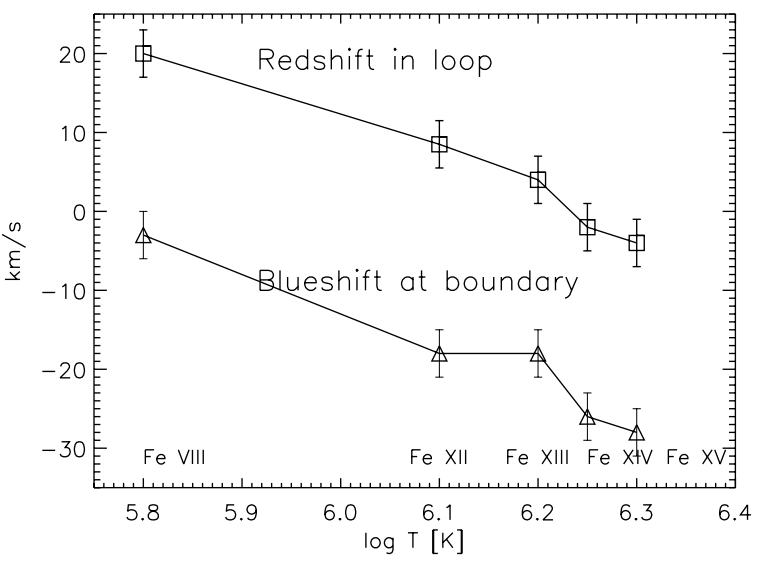

Fig. 3. Dopplershifts measured outside and inside the eastern boundary of the AR observation at 02:54 UT on 2006 December 2.

in a sharp boundary between the closed "hot" (3 MK) loops and the "cooler" (1 MK) extended loops that are well above the "hot" loops. The blue-shifts form as new concentrations emerge, as is clear from the observation of 2006 December 3. Blueshifts are stronger in the hotter lines, typically $5-20 \mathrm{~km} \mathrm{~s}^{-1}$ in Fe XII and $10-30 \mathrm{~km} \mathrm{~s}^{-1}$ in Fe XV. Non-thermal widths are about $50-60 \mathrm{~km} \mathrm{~s}^{-1}$ in Fe XII, which means that there are components in the line that are blueshifted by up to $40 \mathrm{~km} \mathrm{~s}^{-1}$. The blueshifts are located in the regions of lowest density and radiance. The EIS sensitivity is high enough so that hundreds of counts in the stronger lines are still present in these faint areas. Interestingly, regions with strong blueshifts always seem to have the highest non-thermal broadening associated with them (the contrary does not hold). As an example, two regions from the observation of 2006 December 2 at 02:54 UT were selected. The first is on a blue-shifted region in the eastern boundary, at coordinates $X, Y=58,-167$. The second on a region just 7 " westward, where the legs of the low-lying loops are present. The resulting shifts are shown in Fig. 3.

The legs of the 1 MK loops are clearly visible in TRACE and in the EIS TR lines (Fe VIII). Their full extent is only visible in TRACE. The "hot" and "cool" loops are rooted in the same magnetic fragments but at distinct locations. This is clearly shown in Figs. 4 and 5, where the regions of the following (east) and leading (west) polarities are shown.

\section{Discussion and conclusions}

For the first time, EIS observations clearly show a persistent pattern of Dopplershifts in coronal lines across an active region. Despite the low temporal cadence of these EIS observations, the persistence of these shifts is unlikely to be a coincidence. Also, with the help of the high-resolution TRACE observations, it is clear that, for the majority of loops, redshifts occurr in both legs. As already known, the redshifts are greater in cooler lines, near the legs of the loops. It is also clear, for the first time, that blueshifts are located in boundary sharp regions and that shifts are higher in higher-temperature coronal lines. The strongest blueshifts are in low-density regions, which explains why they are so difficult to observe. The blue-shifted regions have large non-thermal withds in coronal lines, larger near the base of the corona.

As interpreted by some authors, it is possible that these redshifts are associated with real downflows. One puzzling issue is the lack of signatures of any upflows in either leg of the loops. Understanding how this occurs could be the key to explain the 

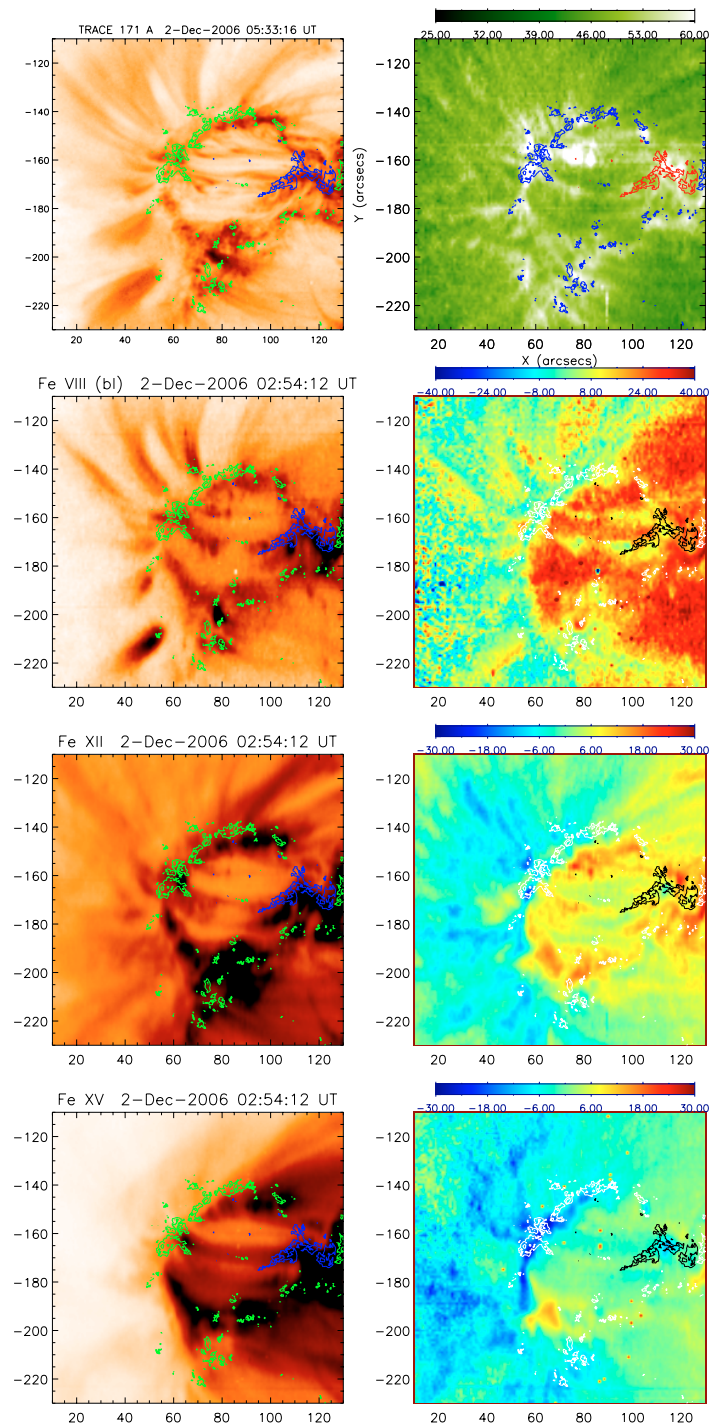

Fig. 4. An enlarged view of the eastern part of the AR as observed on 2006 December 2. In the top, TRACE $171 \AA$ image and nonthermal widths $\left(\mathrm{km} \mathrm{s}^{-1}\right)$ from Fe XII. Then, radiances and Dopplergrams $\left(\mathrm{km} \mathrm{s}^{-1}\right)$ in Fe VIII, Fe XII, Fe XV. Contours from Stokes- $V$ images are superimposed.

mystery of widespread redshifts in transition region lines on the Sun. Detailed forward modelling, using all the measured coronal plasma parameters (flows, densities, temperatures) and timedependent ionization will shed some further light on this issue. It is also possible that the observed blueshifts are related to the dissipation of the 5- and 3-m oscillation waves observed in sunspots and that indicate real upflows in open field regions, as suggested by Marsch et al. (2004).

Interestingly, there are some similarities between this pattern of Dopplershifts/widths and what is observed by CDS during the gradual phase of two ribbon flares (cf. Del Zanna et al. 2006b). There, arcades of cooling plasma are continuously forming, while "gentle" blueshifts (seen in "hot" lines) are located in sharp boundaries outside the arcade. In those cases, the blueshifts are commonly interpreted as the signature of heating/chromospheric evaporation during reconnection. The reconnected loops would then appear and plasma would drain. It is speculated here that the continuous flux emergence and braiding by photospheric motion at the locations of the strongest magnetic fields induces a change in the connectivity to form the
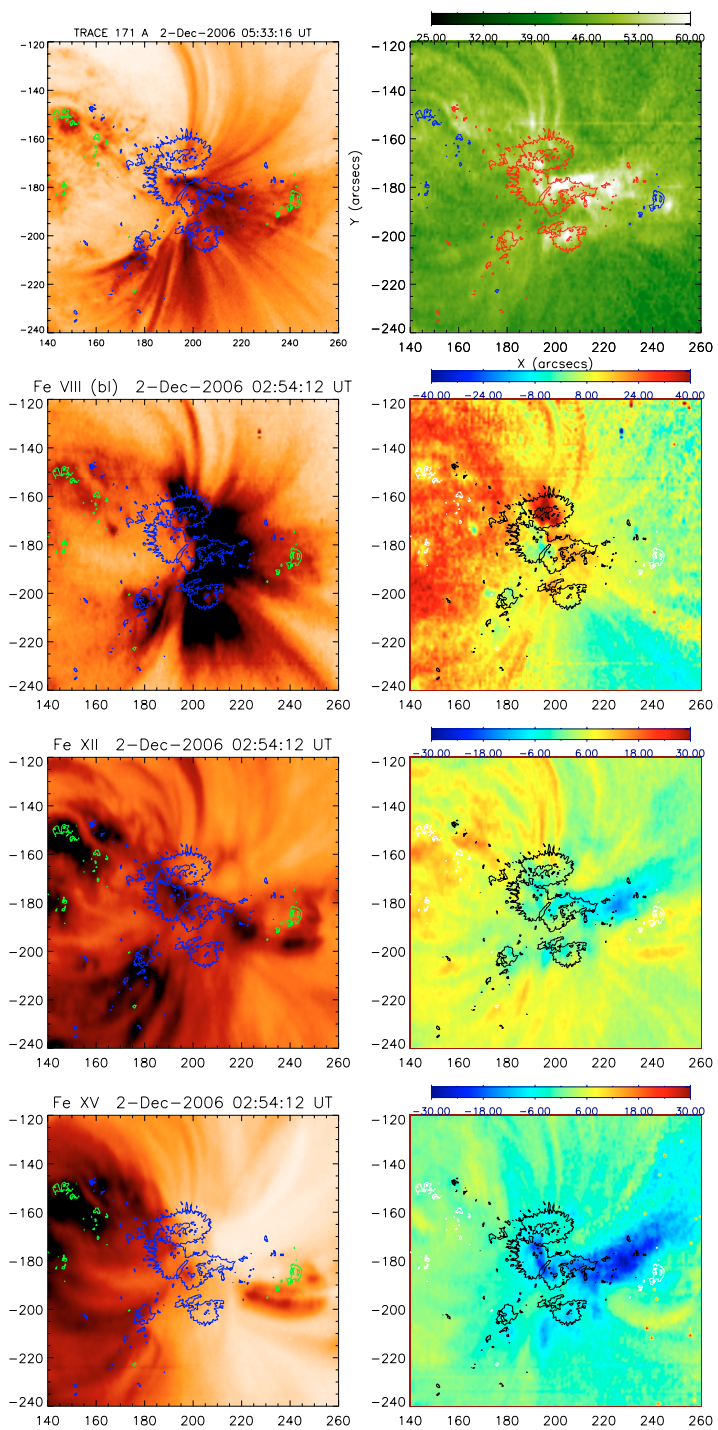

Fig. 5. Same as Fig. 4, but on the the leading sunspot areas.

lower "hot" loops. Detailed analysis and comparison with theoretical modelling will follow, to see if this is a viable hypothesis.

Acknowledgements. Support from STFC (AF) is acknowledged. Hinode is a Japanese mission developed and launched by ISAS/JAXA, with NAOJ as domestic partner and NASA and STFC (UK) as international partners. It is operated by these agencies in co-operation with ESA and NSC (Norway). The SOHO, TRACE, and ESA Hinode data centres were used for this work.

\section{References}

Achour, H., Brekke, P., Kjeldseth-Moe, O., \& Maltby, P. 1995, ApJ, 453, 945 Brynildsen, N., Brekke, P., Fredvik, T., et al. 1998, Sol. Phys., 179, 279 Culhane, J. L., Harra, L. K., James, A. M., et al. 2007, Sol. Phys., 60 Del Zanna, G. 2007, in First Science results from Hinode, ASPC Del Zanna, G., \& Mason, H. E. 2003, A\&A, 406, 1089 Del Zanna, G., Mason, H. E., \& Cirtain, J. 2006a, in ESA SP-617 Del Zanna, G., Schmieder, B., Mason, H., et al. 2006b, Sol. Phys., 239, 173 Doschek, G. A., Mariska, J., Warren, H. P., et al. 2007, ApJ, 667, L109 Gurman, J. B., \& Athay, R. G. 1983, ApJ, 273, 374

Marsch, E., Wiegelmann, T., \& Xia, L. D. 2004, A\&A, 428, 629 Schrijver, C. J. 2001, Sol. Phys., 198, 325

Schrijver, C. J., Title, A. M., Berger, T. E., et al. 1999, Sol. Phys., 187, 261 Winebarger, A. R., Warren, H., van Ballegooijen, A., et al. 2002, ApJ, 567, L89 Young, P. R., Del Zanna, G., Mason, H. E., et al. 2007a, PASJ, 59, 857 Young, P. R., Del Zanna, G., Mason, H. E., et al. 2007b, PASJ, 59, 727 Zwaan, C. 1985, Sol. Phys., 100, 397 